\title{
Theory of defect production in nonlinear quench across a quantum critical point
}

\author{
Shreyoshi Mondal ${ }^{1}$, K. Sengupta ${ }^{1}$, and Diptiman Sen ${ }^{2}$ \\ ${ }^{1}$ T.C.M.P. Division, Saha Institute of Nuclear Physics, 1/AF Bidhannagar, Kolkata 700 064, India \\ ${ }^{2}$ Center for High Energy Physics, Indian Institute of Science, Bangalore, 560 012, India
}

(Dated: November 29, 2018)

\begin{abstract}
We study defect production in a quantum system subjected to a nonlinear power law quench which takes it either through a quantum critical or multicritical point or along a quantum critical line. We elaborate on our earlier work [D. Sen, K. Sengupta, S. Mondal, Phys. Rev. Lett. 101, 016806 (2008)] and present a detailed analysis of the scaling of the defect density $n$ with the quench rate $\tau$ and exponent $\alpha$ for each of the above-mentioned cases. We also compute the correlation functions for defects generated in nonlinear quenches through a quantum critical point and discuss the dependence of the amplitudes of such correlation functions on the exponent $\alpha$. We discuss several experimental systems where these theoretical predictions can be tested.

PACS numbers: $73.43 . \mathrm{Nq}, 05.70 . \mathrm{Jk}, 64.60 . \mathrm{Ht}, 75.10 . \mathrm{Jm}$
\end{abstract}

\section{INTRODUCTION}

Quantum phase transitions have been widely studied in different systems for several years $\stackrel{1}{1}$. Such transitions occur when the ground state of a quantum system changes due to the variation of some system parameter such as pressure $^{2}$, doping ${ }^{3}$ or magnetic field ${ }^{4}$. More recently, non-equilibrium physics around such critical points has also been studied $\stackrel{5,6}{6}$. In particular, quench dynamics through quantum critical points has been a subject of intense theoretical study in recent years. Such a dynamics involves the time evolution of a parameter $\lambda \equiv \lambda(t)$ in the Hamiltonian of the system which carries it through a quantum critical point, characterized by the correlation length exponent $\nu$ and the dynamical critical exponent $z$, at $\lambda=\lambda_{c}$. Since the energy gaps between the ground and the first excited states vanish at the quantum critical point, the dynamics of the system necessarily becomes non-adiabatic in a finite region around this point even for an arbitrarily slow quench. This leads to the failure of the system to follow the instantaneous ground state. As a result defects are produced ${ }^{7,8,9}$. Most of the initial studies of defect production in quench dynamics for various quantum systems have been restricted to the case of a linear quench $\lambda(t) \equiv \lambda_{0} t / \tau$, where $\tau^{-1}$ is the quench rate $e^{6,10,11,12,13,14,15,16,17,18,19,20,21,22}$. It is well known that for a slow linear quench, the defect density $n \sim \tau^{-d \nu /(z \nu+1)}$ where $d$ is the dimension of the system 23,24 . More recently, nonlinear power law quenches characterized by $\lambda(t)=\lambda_{0}|t / \tau|^{\alpha} \operatorname{sign}(t)$, where $\alpha$ denotes the power law exponent and sign is the signum function, have also been studied 25,26 . In particular, it has been shown in Ref. 25, that if, during the quench, the critical point is reached at time $t=0\left[t=t_{0} \neq 0\right]$, then the defect density $n$ for such a quench process scales as $n \sim \tau^{-d \alpha \nu /(\alpha z \nu+1)}\left[n \sim\left(\alpha g^{(\alpha-1) / \alpha} / \tau\right)^{\nu d /(z \nu+1)}\right.$, where $g$ is a non-universal constant.]

On the experimental side, trapped ultracold atoms in optical lattices have provided ways to realize many interacting quantum systems with a variety of low temperature phases separated by quantum critical points 27,28 .
These systems provide an easy access to non-equilibrium dynamics of its constituent atoms and hence provide ideal experimental test beds for quench related studies. Defect production has already been studied experimentally for a spin-1 Bose condensate ${ }^{29}$. However, a detailed experimental study of nonlinear quench dynamics has not been undertaken so far.

In this paper we study defect production due to nonlinear power law quenches in quantum critical systems. Our main results are the following. First, we elaborate on the work of Ref. 25 and provide a detailed derivation of the scaling laws of the defect densities mentioned above. Second, we extend the scaling law for defect production through multicritical points, as studied for a linear quench in Ref. 21, to nonlinear quenches. Third, motivated by the work in Ref. 20, we derive scaling laws for defect densities produced during a nonlinear quench when the system is taken along a gapless line during the quench. Our results extend those in Ref. 21 and 20, and reproduce them as special cases. Fourth, taking the onedimensional Kitaev model as a specific system, we compute the correlation functions for defects produced during a nonlinear quench. We also provide a general model independent discussion of the behavior of such correlation functions. Finally, we present a detailed discussion of possible experimental systems where these theoretical results may be tested.

The organization of the paper is as follows. In Sec. III we provide detailed derivations for the scaling laws of defect density produced during a nonlinear quench. This is followed, in Sec. III by a computation of the defect correlation functions. Next, in Sec. [V] we provide numerical studies to corroborate our analytical results. In Sec. V] we discuss possible experimental systems where the scaling laws derived in Sec. II can be tested. Finally we conclude in Sec. VI 


\section{DEFECT PRODUCTION RATE IN A NONLINEAR QUENCH}

The density of defects produced in a quench process depends crucially on the nature of the phases that the system passes through during the quench. Such processes can therefore be broadly classified into three types. First, the system may pass from one gapped phase to another through an intermediate gapless critical or multicritical point. Second, the system may move along a gapless critical line in the parameter space so that at each point on that line the gap vanishes at a fixed and unique momentum 19,20 . Third, the quench may take the system from a gapped phase to another through a gapless hypersurface in parameter space as well as in momentum space 18 . In what follows, we will study defect production during nonlinear quench of the first two types in Secs. II A, IIB and IIC. An analogous study for the third case, where the system passes through a hypersurface in momentum space, is beyond the scope of the present work.

\section{A. Quench dynamics from one gapped phase to another}

We start with the model Hamiltonian for a $d$ dimensional system

$$
\begin{aligned}
H(t) & =\sum_{\vec{k}} \psi_{\vec{k}}^{\dagger} H_{\vec{k}}(t) \psi_{\vec{k}} \\
H_{\vec{k}}(t) & =(\lambda(t)+b(\vec{k})) \tau_{3}+\Delta(\vec{k}) \tau_{+}+\Delta^{*}(\vec{k}) \tau_{-},
\end{aligned}
$$

where $b(\vec{k})$ and $\Delta(\vec{k})$ are model-dependent functions, $\tau_{i}$ denote usual Pauli matrices, $\lambda(t)=\lambda_{0}|t / \tau|^{\alpha} \operatorname{sign}(t)$ is the quench parameter where $\alpha=1$ implies linear quench, and $\psi_{\vec{k}}=\left(c_{1 \vec{k}}, c_{2 \vec{k}}\right)$ represents the fermionic operators. Such a Hamiltonian is known to represent several one- and twodimensional spin models such as the Ising ${ }^{1}$, the $X Y^{14}$ and the extended Kitaev model 18,31,32. The instantaneous energies of the Hamiltonian given by Eq. (1) are given by

$$
E(\vec{k})= \pm \sqrt{(\lambda(t)+b(\vec{k}))^{2}+|\Delta(\vec{k})|^{2}}
$$

These energy levels touch each other at $t=t_{0}$ and $\vec{k}=\vec{k}_{0}$, so that $|\Delta(\vec{k})| \sim\left|\vec{k}-\vec{k}_{0}\right|$ and $\left|t_{0}\right|=\tau\left|b\left(\vec{k}_{0}\right) / \lambda_{0}\right|^{1 / \alpha}=$ $\tau g^{1 / \alpha}$, where $g=\left|b\left(\vec{k}_{0}\right) / \lambda_{0}\right|$ is a non-universal modeldependent parameter. At this point the energy levels cross and we have a quantum critical point with $\nu=z=$ 1. Note that the critical point is reached at $t=0$ only if $b\left(\vec{k}_{0}\right)$ vanishes.

Let us first consider the case where $b\left(\vec{k}_{0}\right)=0$ so that the system passes through the critical point at $t=0$. In what follows, we shall assume that $|\Delta(\vec{k})| \sim\left|\vec{k}-\vec{k}_{0}\right|$ and $b(\vec{k}) \sim\left|\vec{k}-\vec{k}_{0}\right|^{z_{1}}$ at the critical point, where $z_{1} \geq 1$ so that
$E \sim\left|\vec{k}-\vec{k}_{0}\right|$ and $z=1$. In the rest of the analysis, we set $\hbar=1$, and scale $t \rightarrow t \lambda_{0}, \tau \rightarrow \tau \lambda_{0}, \Delta(\vec{k}) \rightarrow \Delta(\vec{k}) / \lambda_{0}$, and $b(\vec{k}) \rightarrow b(\vec{k}) / \lambda_{0}$.

We begin by observing that the ground state of the system must be $\left(c_{1 \vec{k}}, c_{2 \vec{k}}\right)=(1,0)[(0,1)]$ at the beginning [end] of the quench at $t=-\infty[\infty]$. Thus the probability of defect formation, i.e, the probability for the system to be in the excited state at the end of the quench for a given state $|\vec{k}\rangle$ must be given by

$$
p_{\vec{k}}=\lim _{t \rightarrow \infty}\left|c_{1 \vec{k}}(t)\right|^{2} .
$$

The density of these defects is thus given by

$$
n=\lim _{\mathrm{t} \rightarrow \infty} \int_{\mathrm{BZ}} \frac{d^{d} k}{(2 \pi)^{d}}\left|c_{1 \vec{k}}(t)\right|^{2},
$$

where $\int_{\mathrm{BZ}}$ denotes integration over the Brillouin zone.

To obtain $p_{\vec{k}}$, we study the time evolution of the system which is governed by the Schrödinger equation $i \partial \psi_{\vec{k}} / \partial t=$ $H_{\vec{k}} \psi_{\vec{k}} ;$ this leads to the following equations,

$$
\begin{aligned}
& i \dot{c}_{1 \vec{k}}=\left(|t / \tau|^{\alpha} \operatorname{sign}(t)+b(\vec{k})\right) c_{1 \vec{k}}+\Delta(\vec{k}) c_{2 \vec{k}}, \\
& i \dot{c}_{2 \vec{k}}=-\left(|t / \tau|^{\alpha} \operatorname{sign}(t)+b(\vec{k})\right) c_{2 \vec{k}}+\Delta^{*}(\vec{k}) c_{1 \vec{k}},
\end{aligned}
$$

where we have kept the time dependence of $c_{1 \vec{k}(2 \vec{k})}(t)$ implicit, and $\dot{c}_{1 \vec{k}(2 \vec{k})}(\vec{k}) \equiv \partial_{t} c_{1 \vec{k}(2 \vec{k})}$. To solve these equations, we define

$$
\begin{aligned}
& c_{1 \vec{k}}^{\prime}=c_{1 \vec{k}} e^{i \int^{t} d t^{\prime}\left(\left|t^{\prime} / \tau\right|^{\alpha} \operatorname{sign}\left(t^{\prime}\right)+b(\vec{k})\right)} \\
& c_{2 \vec{k}}^{\prime}=c_{2 \vec{k}} e^{-i \int^{t} d t^{\prime}\left(\left|t^{\prime} / \tau\right|^{\alpha} \operatorname{sign}\left(t^{\prime}\right)+b(\vec{k})\right)} .
\end{aligned}
$$

Then substituting Eq. (6) in Eq. (5) and eliminating $c_{2 \vec{k}}^{\prime}$ from the resulting equations, we get

$$
\begin{aligned}
\ddot{c}_{1 \vec{k}}^{\prime} & -2 i\left[|t / \tau|^{\alpha} \operatorname{sign}(t)+b(\vec{k})\right] \dot{c}_{1 \vec{k}}^{\prime} \\
& +|\Delta(\vec{k})|^{2} c_{1 \vec{k}}^{\prime}=0 .
\end{aligned}
$$

Now we scale $t \rightarrow t \tau^{\alpha /(\alpha+1)}$ so that Eq. (7) becomes

$$
\begin{gathered}
\ddot{c}_{1 \vec{k}}^{\prime}-2 i\left[|t|^{\alpha} \operatorname{sign}(t)+b(\vec{k}) \tau^{\alpha /(\alpha+1)}\right] \dot{c}_{1 \vec{k}}^{\prime} \\
+|\Delta(\vec{k})|^{2} \tau^{2 \alpha /(\alpha+1)} c_{1 \vec{k}}^{\prime}=0 .
\end{gathered}
$$

From Eq. (8) we immediately note that since $c_{1 \vec{k}}$ and $c_{1 \vec{k}}^{\prime}$ differ only by a phase factor, $p_{\vec{k}}$ must be given by

$$
p_{\vec{k}}=\lim _{t \rightarrow \infty}\left|c_{1 \vec{k}}^{\prime}(t)\right|^{2}=f\left[b(\vec{k}) \tau^{\frac{\alpha}{\alpha+1}},|\Delta(\vec{k})|^{2} \tau^{\frac{2 \alpha}{\alpha+1}}\right],(9)
$$

where $f$ is a function whose analytical form is not known for $\alpha \neq 1$. Nevertheless, we note that for a slow quench (large $\tau$ ), $p_{\vec{k}}$ becomes appreciable only when the instantaneous energy gap, as obtained from Eq. (2), becomes small at some point of time during the quench. Consequently, $f$ must vanish when either of its arguments are 
large: $f(\infty, a)=f(a, \infty)=0$ for any value of $a$. Thus for a slow quench (large $\tau$ ), the defect density $n$ is given by

$$
n=\int_{\mathrm{BZ}} \frac{d^{d} k}{(2 \pi)^{d}} f\left[b(\vec{k}) \tau^{\frac{\alpha}{\alpha+1}},|\Delta(\vec{k})|^{2} \tau^{\frac{2 \alpha}{\alpha+1}}\right],
$$

and receives its main contribution from values of $f$ near $\vec{k}=\vec{k}_{0}$ where both $b(\vec{k})$ and $\Delta(\vec{k})$ vanish. Thus one obtains, after extending the range of momentum integration to $\infty$,

$$
n \simeq \int \frac{d^{d} k}{(2 \pi)^{d}} f\left[\left|\vec{k}-\vec{k}_{0}\right|^{z_{1}} \tau^{\frac{\alpha}{\alpha+1}} ;\left|\vec{k}-\vec{k}_{0}\right|^{2} \tau^{\frac{2 \alpha}{\alpha+1}}\right] .
$$

Now scaling $\vec{k} \rightarrow\left(\vec{k}-\vec{k}_{0}\right) \tau^{\alpha /(\alpha+1)}$, we find that

$$
\begin{aligned}
n & =\tau^{-\frac{d \alpha}{\alpha+1}} \int \frac{d^{d} k}{(2 \pi)^{d}} f\left(|\vec{k}|^{z_{1}} \tau^{\alpha\left(1-z_{1}\right) /(\alpha+1)} ;|\vec{k}|^{2}\right) \\
& \simeq \tau^{-\frac{d \alpha}{\alpha+1}} \int \frac{d^{d} k}{(2 \pi)^{d}} f\left(0 ;|\vec{k}|^{2}\right) \sim \tau^{-\frac{d \alpha}{\alpha+1}},
\end{aligned}
$$

where in arriving at the last line, we have used $z_{1}>1$ and $\tau \rightarrow \infty$. (If $z_{1}=1$, the integral in the first line is independent of $\tau$, so the scaling argument still holds). Note that for $\alpha=1$, Eq. (12) reduces to its counterpart for a linear quench $\frac{23}{}$. It turns out that the case $z_{1}<1$ deserves a detailed discussion which we defer till Sec. IIB.

Next we generalize our results for a critical point with arbitrary values of $\nu$ and $z$. To this end, we consider a generic time-dependent Hamiltonian $H_{1}[t] \equiv H_{1}[\lambda(t)]$, whose states are labeled by $|\vec{k}\rangle$ and $|0\rangle$ denotes the ground state. If there is a second order phase transition, the basis states change continuously with time during this evolution and can be written as

$$
|\psi(t)\rangle=\sum_{\vec{k}} a_{\vec{k}}(t)|\vec{k}[\lambda(t)]\rangle .
$$

The defect density can then be obtained in terms of these coefficients $a_{\vec{k}}(t)$ as

$$
n=\sum_{\vec{k} \neq 0}\left|a_{\vec{k}}(t \rightarrow \infty)\right|^{2} .
$$

Following the analysis in Ref. 23, one can then obtain an expression for the defect density $n$ as

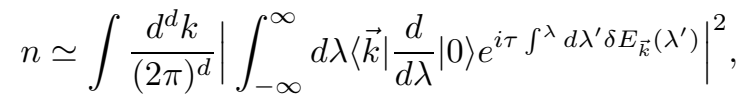

where $\delta E_{\vec{k}}(\lambda)=E_{\vec{k}}(\lambda)-E_{0}(\lambda)$ are the instantaneous excitation energies, and we have replaced the sum over $\vec{k}$ by a $d$-dimensional momentum integral. We note, following Ref. 23, that near a critical point,

$$
\delta E_{\vec{k}}(\lambda)=\Delta F\left(\Delta /\left|\vec{k}-\vec{k}_{0}\right|^{z}\right),
$$

where $\Delta$ is the energy gap, $z$ is the dynamical critical exponent, and $F(x) \sim 1 / x$ for large $x$. Also, since the quench term vanishes at the critical point, $\Delta \sim|\lambda|^{\alpha z \nu}$ for a nonlinear quench, one can write

$$
\delta E_{\vec{k}}(\lambda)=|\lambda|^{\alpha z \nu} F^{\prime}\left(|\lambda|^{\alpha z \nu} /\left|\vec{k}-\vec{k}_{0}\right|^{z}\right),
$$

where $F^{\prime}(x) \sim 1 / x$ for large $x$. Further, one has $\left\langle\vec{k}\left|\frac{d}{d \Delta}\right| 0\right\rangle=\left|\vec{k}-\vec{k}_{0}\right|^{-z} G\left(\Delta /\left|\vec{k}-\vec{k}_{0}\right|^{z}\right)$ near a critical point, where $G(0)$ is a constant. This allows us to write

$$
\left\langle\vec{k}\left|\frac{d}{d \lambda}\right| 0\right\rangle=\frac{\lambda^{\alpha z \nu-1}}{\left|\vec{k}-\vec{k}_{0}\right|^{z}} G^{\prime}\left(\lambda^{\alpha z \nu} /\left|\vec{k}-\vec{k}_{0}\right|^{z}\right),
$$

where $G^{\prime}(0)$ is a constant 1 .23 . Substituting Eqs. (17) and (18) in Eq. (15) and changing the integration variables to $\eta=\tau^{\alpha \nu /(\alpha z \nu+1)}\left|\vec{k}-\vec{k}_{0}\right|$ and $\xi=\left|\vec{k}-\vec{k}_{0}\right|^{-1 /(\alpha \nu)} \lambda$, we find that

$$
n \simeq C \tau^{-\alpha \nu d /(\alpha z \nu+1)},
$$

where $C$ is a non-universal number independent of $\tau$.

Next we focus on the case where the quench term does not vanish at the quantum critical point for $\vec{k}=\vec{k}_{0}$. We again consider the Hamiltonian $H_{\vec{k}}(t)$ in Eq. (1), but now assume that the critical point is reached at $t=t_{0} \neq 0$. This renders our previous scaling argument invalid since $\Delta\left(\vec{k}_{0}\right)=0$ but $b\left(\vec{k}_{0}\right) \neq 0$. In this situation, $\left|t_{0} / \tau\right|=g^{1 / \alpha}$ so that the energy gap $\delta E$ may vanish at the critical point for $\vec{k}=\vec{k}_{0}$. We now note that the most important contribution to the defect production comes from times near $t_{0}$ and from momenta near $k_{0}$. Hence we expand the diagonal terms in $H_{\vec{k}}(t)$ about $t=t_{0}$ and $\vec{k}=\vec{k}_{0}$ to obtain

$$
\begin{array}{r}
H^{\prime}(t)=\sum_{\vec{k}} \psi^{\dagger}(\vec{k})\left[\left\{\alpha g^{(\alpha-1) / \alpha}\left(\frac{t-t_{0}}{\tau}\right)+b^{\prime}(\delta \vec{k})\right\} \tau_{3}\right. \\
\left.+\Delta(\vec{k}) \tau_{+}+\Delta^{*}(\vec{k}) \tau_{-}\right] \psi(\vec{k}), \quad(20)
\end{array}
$$

where $b^{\prime}(\delta \vec{k})$ represents all the terms in the expansion of $b(\vec{k})$ about $\vec{k}=\vec{k}_{0}$, and we have neglected all terms

$$
\begin{aligned}
R_{n}= & (\alpha-n+1)(\alpha-n+2) \ldots(\alpha) \\
& \times g^{(\alpha-n) / \alpha}\left|\left(t-t_{0}\right) / \tau\right|^{n} \operatorname{sign}(t) / n !
\end{aligned}
$$

for $n>1$ in the expansion of $\lambda(t)$ about $t_{0}$. We shall justify neglecting these higher order terms shortly.

Eq. (201) describes a linear quench of the system with $\tau_{\text {eff }}(\alpha)=\tau /\left(\alpha g^{(\alpha-1) / \alpha}\right)$. Hence one can use the wellknown results of Landau-Zener dynamics ${ }^{30}$ to write an expression for the defect density,

$$
n=\int_{\mathrm{BZ}} \frac{d^{d} k}{(2 \pi)^{d}} p_{\vec{k}}=\int_{\mathrm{BZ}} \frac{d^{d} k}{(2 \pi)^{d}} \exp \left[-\pi|\Delta(\vec{k})|^{2} \tau_{\mathrm{eff}}(\alpha)\right](22)
$$

For a slow quench, the contribution to $n$ comes from $\vec{k}$ near $\vec{k}_{0}$; hence

$$
n \sim \tau_{\mathrm{eff}}(\alpha)^{-d / 2}=\left(\alpha g^{(\alpha-1) / \alpha} / \tau\right)^{d / 2} .
$$


Note that for the special case $\alpha=1$, we get back the familiar result $n \sim \tau^{-d / 2}$, and the dependence of $n$ on the non-universal constant $g$ vanishes. Also, since the quench is effectively linear, we can use the results of Ref. 23 to find the scaling of the defect density when the critical point at $t=t_{0}$ is characterized by arbitrary $\nu$ and $z$,

$$
n \sim\left(\alpha g^{(\alpha-1) / \alpha} / \tau\right)^{\nu d /(z \nu+1)} .
$$

Next we justify neglecting the higher order terms $R_{n}$. We note that significant contributions to $n$ come at times $t$ when the instantaneous energy levels of $H^{\prime}(t)$ in Eq. (20) for a given $\vec{k}$ are close to each other, i.e., $\left(t-t_{0}\right) / \tau \sim \Delta(\vec{k})$. Also, for a slow quench, the contribution to the defect density is substantial only when $p_{\vec{k}}$ is significant, namely, when $|\Delta(\vec{k})|^{2} \sim 1 / \tau_{\text {eff }}(\alpha)$. Using these arguments, we see that

$$
\begin{aligned}
R_{n} / R_{n-1} & =(\alpha-n+1) g^{-1 / \alpha}\left(t-t_{0}\right) /(n \tau) \\
& \sim(\alpha-n+1) /(n \sqrt{\tau}) .
\end{aligned}
$$

Thus we find that all higher order terms $R_{n>1}$, which were neglected in arriving at Eq. (23), are unimportant in the limit of slow quench (large $\tau$ ).

The scaling relations for the defect density $n$ given by Eqs. (19) and (24) represent the central results of this section. For such power law quenches, unlike their linear counterpart, $n$ depends crucially on whether or not the quench term vanishes at the critical point. For quenches which do not vanish at the critical point, $n$ scales with the same exponent as that of a linear quench, but is characterized by a modified non-universal effective rate $\tau_{\text {eff }}(\alpha)$. If, however, the quench term vanishes at the critical point, we find that $n$ scales with a novel $\alpha$-dependent exponent $\alpha d \nu /(\alpha z \nu+1)$. For $\alpha=1, \tau_{\text {eff }}(\alpha)=\tau$ and $\alpha d \nu /(\alpha z \nu+1)=d \nu /(z \nu+1)$; hence both Eqs. (19) and (24) reproduce the well-known defect production law for linear quenches as a special case $\underline{23}$. We note that the scaling of $n$ will show a cross-over between the expressions given in Eqs. (19) and (24) near some value of $\tau=\tau_{0}$ which can be found by equating these two expressions; this yields $\tau_{0} \sim\left|b\left(\vec{k}_{0}\right)\right|^{-z \nu-1 / \alpha}$. For $\alpha>1$, the scaling law will thus be given by Eq. (19) (Eq. (24)) for $\tau \ll(\gg) \tau_{0}$. We also note here that the results of this section assumes that the system passes from one gapped phase to another through a critical point and do not apply to quenches which take a system along a critical line 18.19. We shall deal with this case in Sec. [IC]

\section{B. Quench dynamics through a multicritical point}

In this section, we will consider the effect of a nonlinear quench in a system of the form given in Eq. (11), except that we now take

$$
b(\vec{k}) \sim\left|\vec{k}-\vec{k}_{0}\right|^{z_{1}}, \quad \text { and } \quad \Delta(\vec{k}) \sim\left|\vec{k}-\vec{k}_{0}\right|^{z_{2}},
$$

so that the system passes through the critical point at $t=0$. This will be a generalization of the discussion in the first part of Sec. [IA where we had $z_{1}>z_{2}$ with $z_{2}=1$. We will see below that a separate analysis is required if $z_{2}>z_{1}$. As discussed recently in Ref. 21, such a condition arises at the multicritical point of a onedimensional spin-1/2 XY model in a transverse field; in that model, we find that $z_{1}=2$ and $z_{2}=3$.

We begin our analysis by comparing the diagonal and off-diagonal terms in Eq. (11). From general considerations, it is clear that defects are mainly produced when both $|t / \tau|^{\alpha} \operatorname{sign}(t)+b(\vec{k})$ and $|\Delta(\vec{k})|$ are of order 1 or less since this is when the instantaneous energy levels given by Eq. 2 are close to each other. We now consider the forms of $b(\vec{k})$ and $\Delta(\vec{k})$ given in Eq. (26). Two possibilities arise in the limit $\tau \rightarrow \infty$ and $\left|\vec{k}-\vec{k}_{0}\right| \rightarrow 0$.

(i) If $z_{1}>z_{2}$, then $|\Delta(\vec{k})|$ being of order 1 or less implies that $b(\vec{k}) \ll|\Delta(\vec{k})|$, namely, $b(\vec{k}) \ll 1$. In this case, we can ignore the term $b(\vec{k})$ in Eq. (11). This is equivalent to saying that the first argument of the scaling function $f$ in Eq. (9) can be set equal to zero. Following arguments similar to those leading up to Eq. (12), we then see that the defect density scales as

$$
n \sim \tau^{-d \alpha /\left[z_{2}(\alpha+1)\right]}
$$

which is independent of the value of $z_{1}$.

(ii) If $z_{2}>z_{1}$, then $|\Delta(\vec{k})|$ being of order 1 or less implies that $b(\vec{k}) \gg|\Delta(\vec{k})|$, namely, $b(\vec{k}) \gg 1$. Thus $b(\vec{k})$ always remains finite as we approach the critical point and cannot in general be neglected. In order to have $|t / \tau|^{\alpha} \operatorname{sign}(t)+b(\vec{k})$ of order 1 or less, we must therefore have $t \gg 1$. Let us define a time $t_{0}$ as $\left|t_{0} / \tau\right|^{\alpha}=$ $-\operatorname{sign}\left(\mathrm{t}_{0}\right) b(\vec{k})=-\operatorname{sign}\left(\mathrm{t}_{0}\right) \eta\left|\vec{k}-\vec{k}_{0}\right|^{z_{1}}$, where $\eta$ is an arbitrary non-universal constant. Thus

$$
\left|t_{0}\right|=|\eta|^{1 / \alpha} \tau\left|\vec{k}-\vec{k}_{0}\right|^{z_{1} / \alpha} .
$$

In a spirit similar to Eq. (20), we now linearize the function $|t / \tau|^{\alpha} \operatorname{sign}(t)+b(\vec{k})$ near $t=t_{0}$, as $\left(|t / \tau|^{\alpha} \operatorname{sign}(\mathrm{t})-\right.$ $\left.\left|t_{0} / \tau\right|^{\alpha} \operatorname{sign}\left(\mathrm{t}_{0}\right)\right)=\left(t-t_{0}\right) \alpha\left|t_{0} / \tau\right|^{\alpha-1} / \tau$ which, using Eq. (28), is equal to $\left(t-t_{0}\right) / \tau_{\text {eff }}(|\vec{k}| ; \alpha)$ where

$$
\tau_{\text {eff }}(|\vec{k}| ; \alpha) \equiv \tau_{\text {eff }}=\tau|\eta|^{(\alpha-1) / \alpha}\left|\vec{k}-\vec{k}_{0}\right|^{-z_{1}(\alpha-1) / \alpha} / \alpha .
$$

The effective linearized Hamiltonian can be written as

$$
H_{\mathrm{eff}}=\left(\tau_{3}\left(t-t_{0}\right) / \tau_{\mathrm{eff}}+\left|\vec{k}-\vec{k}_{0}\right|^{z_{2}} \tau_{1}\right)
$$

and describes a linear quench with $\tau$ replaced by $\tau_{\text {eff }}(|\vec{k}| ; \alpha)$. The corresponding defect density is therefore given by the Landau-Zener expression in Eq. (22). We find that

$$
p_{\vec{k}} \sim \exp \left[-\pi \tau\left|\vec{k}-\vec{k}_{0}\right|^{\left[2 z_{2}-z_{1}(1-1 / \alpha)\right]}|\eta|^{(\alpha-1) / \alpha} / \alpha\right],
$$


and

$$
n \sim \tau^{-d \alpha /\left[2 z_{2} \alpha+z_{1}(1-\alpha)\right]} .
$$

Note that the defect density obtained in Eq. (32) scales with an exponent which is independent of the nonuniversal coefficient $\eta$.

To generalize these results for models with arbitrary $z_{1}<z_{2}$ and $\nu$, we notice that such models can be described by an effective Hamiltonian $H_{\text {eff }}(\lambda(t))$, where $\lambda(t)=\left(t-t_{0}\right) / \tau_{\text {eff }}(|\vec{k}| ; \alpha)$ and $\tau_{\text {eff }}(|\vec{k}| ; \alpha)$ is given by Eq. (29). This effective Hamiltonian therefore describes a linear quench with a different $\tau_{\text {eff }}$ for each $\vec{k}$ mode and with effective dynamical critical exponent $z_{2}$ and correlation length exponent $\nu$. Thus using the arguments of Ref. 23, we get

$n \simeq \int \frac{d^{d} k}{(2 \pi)^{d}}\left|\int_{-\infty}^{\infty} d \lambda\left\langle\vec{k}\left|\frac{d}{d \lambda}\right| 0\right\rangle e^{i \tau_{\text {eff }}(|\vec{k}| ; \alpha) \int^{\lambda} d \lambda^{\prime} \delta E_{\vec{k}}\left(\lambda^{\prime}\right)}\right|^{2}$,

where $\delta E_{\vec{k}}(\lambda) \simeq|\lambda|^{z_{2} \nu} F^{\prime}\left(|\lambda|^{z_{2} \nu} /|\vec{k}|^{z_{2}}\right)$, and $F^{\prime}(x) \sim$ $1 / x$ for large $x$. Further, one has $\left\langle\vec{k}\left|\frac{d}{d \Delta}\right| 0\right\rangle=$ $|\vec{k}|^{-z_{2}} G\left(\Delta /|\vec{k}|^{z_{2}}\right)$ near a critical point, where $G(0)$ is a constant. Using these relations, one obtains $n \simeq$ $\int d^{d} k \mid \int d \lambda^{\prime} \lambda^{\prime} z_{2} \nu-1 G\left(\lambda^{\prime}\right) \exp \left(\frac{i \tau}{\alpha}|\vec{k}|^{\left[\alpha\left(z_{2} \nu+1\right)+z_{1} \nu(1-\alpha)\right] / \alpha \nu}\right.$ $\left.\int^{\lambda^{\prime}} d \lambda^{\prime \prime} \lambda^{\prime \prime} F\left(\lambda^{\prime \prime}\right)\right)\left.\right|^{2}$, where $\lambda^{\prime}=\lambda /|\vec{k}|^{1 / \alpha \nu}$ and we have set $\eta=1$ without any loss of generality. Then scaling $|\vec{k}| \rightarrow|\vec{k}| \tau^{\alpha \nu /\left[\alpha\left(z_{2} \nu+1\right)+z_{1} \nu(1-\alpha)\right]}$, one finally gets

$$
n \sim \tau^{-d \alpha \nu /\left[\alpha\left(z_{2} \nu+1\right)+z_{1} \nu(1-\alpha)\right]},
$$

which reduces to Eq. (32) for $z_{2} \nu=1$. Note that for Eq. (27), a generalization to models with arbitrary $z_{2} \nu$ is straightforward, and is given by Eq. (19) with $z$ replaced by $z_{2}$.

Eqs. (27), (32) and (34) are the main results of this section. These results generalize those in Sec. IIA to defect production for quenches through arbitrary multicritical points. Note that for $z_{1}=z_{2}$, Eq. (27) and Eq. (32) agree for any value of $\alpha$, giving $n \sim \tau^{-d \alpha /\left[z_{2}(\alpha+1)\right]}$. Further, for the case of linear quenching, $\alpha=1$, these equations agree for any value of $z_{1}$ and $z_{2}$, giving $n \sim$ $\tau^{-d /\left(2 z_{2}\right)}$ which has been recently obtained in Ref. 21 .

\section{Quench dynamics along a gapless line}

Recently quench dynamics in a one-dimensional $X Y$ model in the presence of a spatially modulated transverse magnetic field has been studied in Ref. 20. Such a model is described by the Hamiltonian

$$
\begin{aligned}
H= & -\frac{1}{2} \sum_{j}\left[J\left(\sigma_{j}^{x} \sigma_{j+1}^{x}+\sigma_{j}^{y} \sigma_{j+1}^{y}\right)\right. \\
& \left.+\gamma\left(\sigma_{j}^{x} \sigma_{j+1}^{x}-\sigma_{j}^{y} \sigma_{j+1}^{y}\right)-\left(h-(-1)^{j} \delta\right) \sigma_{j}^{z}\right]
\end{aligned}
$$

where $J$ and $\gamma$ are respectively the strength of and the anisotropy in the nearest neighbor spin-spin interactions, $\sigma^{a}(a=x, y, z)$ denote the Pauli matrices, and $h$ and $\delta$ denote the uniform and alternating components of the magnetic fields respectively. The phase diagram of this model is discussed in detail in Ref. 20. It was pointed out that quenching the anisotropy parameter $\gamma(t)=\gamma_{0} t / \tau$ linearly while sitting at the paramagnetic phase determined by the condition $h^{2}=\delta^{2}+J^{2}$, leads to a time evolution of the system along a gapless line. It was also shown, via mapping this spin model to a system of Majorana fermions by a Jordan-Wigner transformation, that the evolution of the model described by Eq. (35) can be represented by an effective Hamiltonian given by 20

$$
H_{\mathrm{eff}}(k ; t)=\sum_{k} \psi_{k}^{\dagger}\left(\tilde{\gamma}(t) k \tau_{3}+\tilde{J} k^{2} \tau_{1}\right) \psi_{k},
$$

where $\psi_{k}=\left(c_{1 k}, c_{2 k}\right)$ is the usual two component fermionic field, $\tilde{\gamma}(t)=\gamma(t) J / \sqrt{\delta^{2}+J^{2}}$ and $\tilde{J}=$ $J^{2} / \sqrt{\delta^{2}+J^{2}}$. The quench dynamics of this model was studied in Ref. 20 for the linear quench $\gamma(t)=\gamma_{0} t / \tau$ using the Landau-Zener formalism. It was found that the defect density scales as

$$
n \sim \tau^{-1 / 3} .
$$

Note that since for this model $z=\nu=1$, the naive expectation according to the analysis of Sec. IIA is to have $n \sim 1 / \sqrt{\tau}$. This result therefore clearly points out the necessity of extending the analysis of Sec. IIA for quenches along gapless lines in parameter space. In what follows, we shall only restrict ourselves to quenches where the gap vanishes at the same momentum value $k_{0} ; k_{0}=0$ for the present case.

We start with a generic $d$-dimensional model described by a Hamiltonian

$$
H_{\text {eff }}(k ; t)=\sum_{k} \psi_{k}^{\dagger}\left(\lambda(t)|\vec{k}|^{a} \tau_{3}+\Delta_{0}|\vec{k}|^{b} \tau_{1}\right) \psi_{k},
$$

where $\lambda(t)=\lambda_{0}|t / \tau|^{\alpha} \operatorname{sign}(t)$ is the quench parameter, $a$ and $b$ are arbitrary exponents, and we have taken $\vec{k}_{0}=0$ for clarity. Note that $d=a=1$ and $b=2$ corresponds to the $X Y$ model studied in Ref. 20, while $a=0$ and $d=b=1$ corresponds to the one-dimensional Kitaev model studied in Ref. 18. For $a \neq 0$, the system passes along a gapless line during the quench. We study the time evolution of the model in a manner similar to that described in Sec. IIA After some straightforward algebra, one obtains the equation for the evolution of $c_{1 \vec{k}}^{\prime}(t)=c_{1 \vec{k}}(t) \exp \left(i \int^{t} d t^{\prime}\left|t^{\prime} / \tau\right|^{\alpha} \operatorname{sign}\left(t^{\prime}\right)|\vec{k}|^{a}\right)$ as

$$
\ddot{c}_{1 \vec{k}}^{\prime}-2 i|t / \tau|^{\alpha} \operatorname{sign}(t)|\vec{k}|^{a} \dot{c}_{1 \vec{k}}^{\prime}+\Delta_{0}^{2}|\vec{k}|^{2 b} c_{1 \vec{k}}^{\prime}=0 .
$$

Next we define $\tau_{\vec{k}}=\tau / k^{a / \alpha}$ and scale $t \rightarrow t \tau_{\vec{k}}^{\alpha /(\alpha+1)}$ in Eq. (39) to obtain

$$
\begin{aligned}
\ddot{c}_{1 \vec{k}}^{\prime} & -2 i|t|^{\alpha} \operatorname{sign}(t) \dot{c}_{1 \vec{k}}^{\prime} \\
& +|\Delta|_{0}^{2}|\vec{k}|^{2 b-\frac{2 a}{\alpha+1}} \tau^{\frac{2 \alpha}{\alpha+1}} c_{1 \vec{k}}^{\prime}=0 .
\end{aligned}
$$


From Eq. (40), we find that the probability of defect formation for a given momentum $\vec{k}$ must be given by

$$
p_{\vec{k}}=\lim _{t \rightarrow \infty}\left|c_{1 \vec{k}}^{\prime}(t)\right|^{2}=f\left[\Delta_{0}^{2}|\vec{k}|^{2 b-2 a /(\alpha+1)} \tau^{2 \alpha /(\alpha+1)}\right],
$$

where $f[\infty]=0$. The defect density therefore becomes

$$
n \sim \int_{B Z} \frac{d^{d} k}{(2 \pi)^{d}} f\left(\Delta_{0}^{2} \tau^{\frac{2 \alpha}{\alpha+1}}|\vec{k}|^{2 b-\frac{2 a}{\alpha+1}}\right) .
$$

Using the same logic as outlined in Sec. IIA, we scale $|\vec{k}| \rightarrow \tau^{\frac{\alpha}{b(\alpha+1)-a}}|\vec{k}|$ and get

$$
n \sim \tau^{-\frac{d \alpha}{b(\alpha+1)-a}} .
$$

This result generates the scaling of the defect density derived in Ref. $20\left(n \sim \tau^{-d /(2 b-a)}\right)$ for the special case $\alpha=1$, and that of the one-dimensional Kitaev model studied in Ref. 18 for $\alpha=b=z=1$ and $a=0$.

Finally, we generalize the result in Eq. (43) to systems where the energy difference between the ground and excited states vanishes along the gapless line as $\Delta=\lambda^{\alpha c}|\vec{k}|^{a}$. Note that for the quenches treated in Sec. IIA $c=z \nu$. Here, however, since the quench takes place along a gapless line, $c$ need not have the same interpretation and can be system specific. Exactly at the quantum critical point $\lambda=\lambda_{c}$, the energy gap vanishes as $\Delta \sim|\vec{k}|^{b}$. Thus $b$ is to be interpreted as the dynamical scaling exponent $z$ in the present case. Then using the same scaling argument as in Sec. II A we can express the defect density $n$ using Eq. (15). However, in the present case the energy gap scales as

$$
\begin{aligned}
E_{k}(\lambda)-E_{0}(\lambda) & =\Delta F\left(\Delta /|k|^{b}\right), \\
\left\langle\vec{k}\left|\frac{d}{d \Delta}\right| 0\right\rangle & =1 /|k|^{b} G\left(\Delta /|k|^{b}\right),
\end{aligned}
$$

where $F(x)=1 / x$ for large $x$, and $G(0)$ is a constant. Using Eq. (44), we see that

$$
\begin{aligned}
E_{k}(\lambda)-E_{0}(\lambda) & =\lambda^{\alpha c}|\vec{k}|^{a} F\left(\lambda^{\alpha c}|\vec{k}|^{a} /|\vec{k}|^{b}\right), \\
\left\langle\vec{k}\left|\frac{d}{d \lambda}\right| 0\right\rangle & =\left\langle\vec{k}\left|\frac{d}{d \Delta}\right| 0\right\rangle \frac{d \Delta}{d \lambda} \\
& \simeq \frac{\lambda^{\alpha c-1}}{|\vec{k}|^{(b-a)}} G\left(\frac{\lambda^{\alpha c}|\vec{k}|^{a}}{|\vec{k}|^{b}}\right) .
\end{aligned}
$$

Substituting these in Eq. (15), the defect density produced in this system is found to be

$$
\begin{aligned}
n \sim \int \frac{d^{d} k}{(2 \pi)^{d}} & \mid \int_{-\infty}^{\infty} \frac{\lambda^{\alpha c-1}}{|\vec{k}|^{(b-a)}} G\left(\frac{\lambda^{\alpha c}}{|\vec{k}|^{b-a}}\right) \\
& e^{i \tau \int^{\lambda} d \lambda^{\prime} \lambda^{\prime \alpha c}|\vec{k}|^{a} F\left(\lambda^{\prime \alpha c}|\vec{k}|^{a} /|\vec{k}|^{b}\right)} \mid .
\end{aligned}
$$

Defining new variables $\xi=\lambda|\vec{k}|^{(a-b) / \alpha c}$ and $\left|\vec{k}^{\prime}\right|=$ $|\vec{k}| \tau^{\alpha c /[(b-a)+b \alpha c]}$, we get

$$
n \sim \tau^{-d \alpha c /[(b-a)+b \alpha c]} .
$$

Eq. (477) is one of the central results of this work, and it generates all the previous scaling laws for both linear and nonlinear quenches through critical lines and points (but not through multicritical points) as special cases. For $a=0, b=z$ and $c=z \nu$, we recover the scaling law Eq. (19) for a nonlinear quench, whereas for $\alpha=c=1$, we obtain the scaling law derived for a linear quench in Ref. 20.

\section{DEFECT CORRELATION FUNCTIONS}

For the purpose of computation of defect correlation functions, we are going to restrict ourselves to the class of $d$-dimensional models given by $H(t)$ in Eq. (11). As mentioned before, many standard spin models in one and two dimensions can be mapped, via standard Jordan-Wigner transformations $\mathbb{1}^{1}$, to such fermionic models described by $H(t)$. Let us denote the ground and the excited states of $H(t)$ before the quench (at $t=-\infty$ ) by $|0\rangle_{\vec{k}}$ and $|1\rangle_{\vec{k}}$ respectively for a given value of $\vec{k}$. Then the state of the system after the quench (at $t=\infty$ ) is given by$\underline{\underline{18}}$

$$
|\phi\rangle_{\vec{k}}=\sqrt{p_{\vec{k}}}|0\rangle_{\vec{k}}+\sqrt{1-p_{\vec{k}}}|1\rangle_{\vec{k}} .
$$

Using this, one can compute the defect correlation functions for these models. These correlation functions are of two types. They can either vanish at the origin, as in the case of the two-dimensional extended Kitaev model ${ }^{18}$, or can be written as $\underline{10,18}$

$$
\left\langle O_{\vec{r}}\right\rangle=-\delta_{\vec{r}, 0}+C \int_{0}^{2 \pi} d^{d} k f\left[|\Delta(\vec{k})|^{2} \tau^{\frac{2 \alpha}{\alpha+1}}\right] g(\vec{k} \cdot \vec{r}),
$$

where $O_{\vec{r}}=i \psi_{\vec{n}} \psi_{\vec{n}+\vec{r}}, \psi_{\vec{n}}$ denotes the field operators for Majorana fermions, $g(\vec{k} \cdot \vec{r})$ is a system specific function independent of $\tau, C$ denotes a system and dimension specific constant which will be unimportant for subsequent discussions, and we have used Eq. (9) to obtain the value of $p_{\vec{k}}$. Since for a slow quench, $p_{\vec{k}}$ is appreciable only near $\vec{k}=\vec{k}_{0}$, we expand $\Delta(\vec{k})$ about $\vec{k}_{0}$, scale the momentum components $k_{i}^{\prime}=\left(k-k_{0}\right)_{i} \tau^{\alpha /(\alpha+1)}$, and extend the range of integration to $\infty$ to get

$$
\left\langle O_{\vec{r}}\right\rangle=-\delta_{\vec{r}, 0}+\frac{C}{\tau^{d \alpha /(\alpha+1)}} \int_{0}^{\infty} d^{d} k^{\prime} f\left[\left|\vec{k}^{\prime}\right|^{2}\right] g\left(\vec{k}^{\prime} \cdot \vec{r}^{\prime}\right),
$$

where $\vec{r}_{i}^{\prime}=\vec{r}_{i} / \tau^{\alpha /(\alpha+1)}$. Thus we find that quite generally, for the class of models whose defect correlation functions do not vanish at the origin,

$$
\ln \left(1+\left\langle O_{\vec{r}=0}\right\rangle\right)=\ln \left(C^{\prime}\right)-\frac{d \alpha}{\alpha+1} \ln (\tau),
$$

i.e., the logarithm of the deviation of the amplitude of these correlation functions at the origin from -1 is a linear function of $\ln (\tau)$ with a slope of $-d \alpha /(\alpha+1)$. 


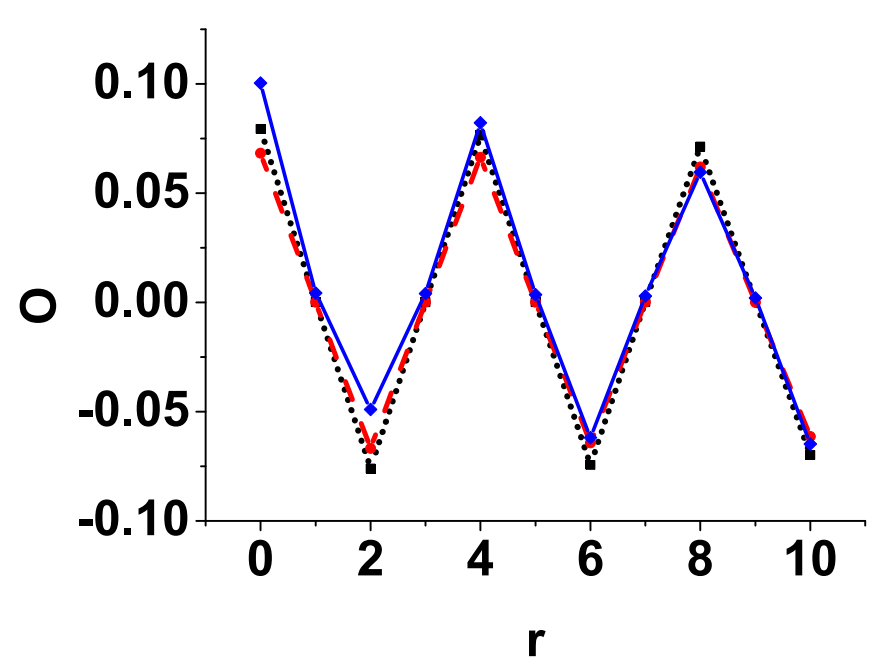

FIG. 1: Plot of $\left\langle O_{r}\right\rangle$ vs $r$ for $\alpha=2$ (black dot-dashed line), $\alpha=3$ (red dashed line), $\alpha=4$ (blue solid line), and $\tau=20$.

We now compute the correlation function for a specific model, namely, the one-dimensional Kitaev model [18,25, 33] which has the Hamiltonian

$$
H=\sum_{i \in \text { even }}\left(J_{1} S_{i}^{x} S_{i+1}^{x}+J_{2} S_{i}^{y} S_{i-1}^{y}\right)
$$

where $J_{1}$ and $J_{2}$ denote the nearest neighbor interaction strengths, and $S_{i}$ denotes the spin at site $i$. Using the standard Jordan-Wigner transformation, this Hamiltonian can be mapped on to a free fermionic Hamiltonian [18, 25, 33.

$$
\begin{aligned}
H & =\sum_{\vec{k}} \psi_{k}^{\dagger} H_{k} \psi_{k}, \quad \text { where } \\
H_{k} & =-2\left(J_{-} \sin (k) \tau_{3}+J_{+} \cos (k) \tau_{2}\right) .
\end{aligned}
$$

Here $J_{ \pm}=J_{1} \pm J_{2}$, and $\psi_{k}=\left(c_{1}(k), c_{2}(k)\right)$ are the fermionic fields. The Hamiltonian is changed in time by varying the parameter $J_{-}$keeping $J_{+}$fixed. The defect correlation function for this model is given by [18]

$$
\left\langle O_{r}\right\rangle=-\delta_{r, 0}+\frac{2}{\pi} \int_{0}^{\pi} d k p_{k} \cos (k r)
$$

Thus we find that the defect correlation functions have the same form as in Eq. (49) with $C=2 / \pi$ and $g=$ $\cos (k r)$. A plot of the correlation function as a function of $r$, sans the $\delta$-function peak at the origin, is shown in Fig. 1 for $\tau=20$ and several representative values of $\alpha$.

\section{NUMERICAL EVALUATION OF DEFECT DENSITIES}

In this section, we provide numerical studies of the onedimensional Ising and Kitaev models to supplement our

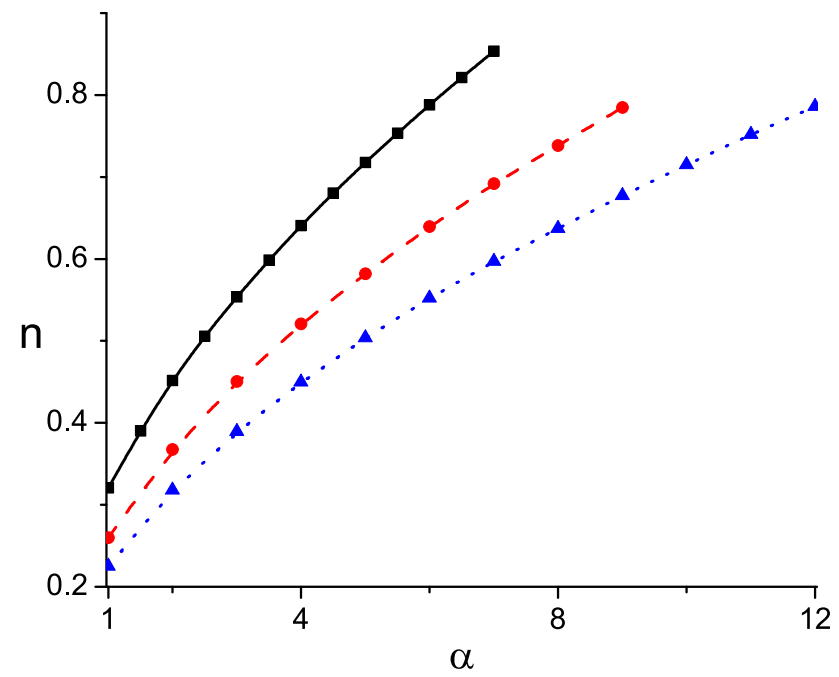

FIG. 2: Variation of the defect density $n$ with the quench exponent $\alpha$ for representative values of $\tau=10$ (black solid line), $\tau=15$ (red dashed line) and $\tau=20$ (blue dotted line). A polynomial fit of the form $n=a \alpha^{b}$ yields exponents which are very close to the theoretical result $1 / 2$ for all values of $\tau$ (see text for details).

analytical results. First we consider the one-dimensional Ising model in a transverse field described by

$$
H_{\text {Ising }}=-J\left(\sum_{i} S_{i}^{z} S_{i+1}^{z}+g \sum_{i} S_{i}^{x}\right),
$$

where $J$ is the strength of the nearest neighbor interaction, and $g=h / J$ is the dimensionless transverse field. In what follows, we shall quench the transverse field as $g(t)=|t / \tau|^{\alpha} \operatorname{sign}(t)$ and compute the density of the resultant defects.

We begin by mapping $H_{\text {Ising }}$ to a system of free fermions via a standard Jordan-Wigner transformation 1

$$
H^{\prime}=-J \sum_{k} \psi_{k}^{\dagger}\left((g-\cos (k)) \tau_{3}+\sin (k) \tau_{1}\right) \psi_{k} .
$$

If the external magnetic field $g$ is varied with time as $g(t)=g_{0}|t / \tau|^{\alpha} \operatorname{sign}(t)$, then the system will go through two quantum critical points at $g=1$ and -1 . The energy gap vanishes at these quantum critical points at $k=$ $k_{0}=0$ and $\pi$. As a result, defects are produced in nonadiabatic regions near these points. For this model, the quantum critical point is at $t=t_{0} \neq 0$ and $z=\nu=1$. Hence, $\tau_{\text {eff }}=\tau / \alpha$ for both the quantum critical points. From Eq. (24), therefore, we expect the defect density produced in this system to be given by $n \sim(\tau / \alpha)^{-1 / 2}$.

To verify this expectation, we numerically solve the Schrödinger equation $i \partial_{t} \psi_{k}=H_{k}(t) \psi_{k}$ and obtain the probability $p_{k}$ for the system to be in the excited state. Finally, integrating over all $k$ within the Brillouin zone, we obtain the defect density $n$ for different values of $\alpha>$ 1 with fixed $\tau$. The plot of $n$ as a function of $\alpha$ for $\tau=10,15$ and 20 is shown in Fig. 2. A fit to these 


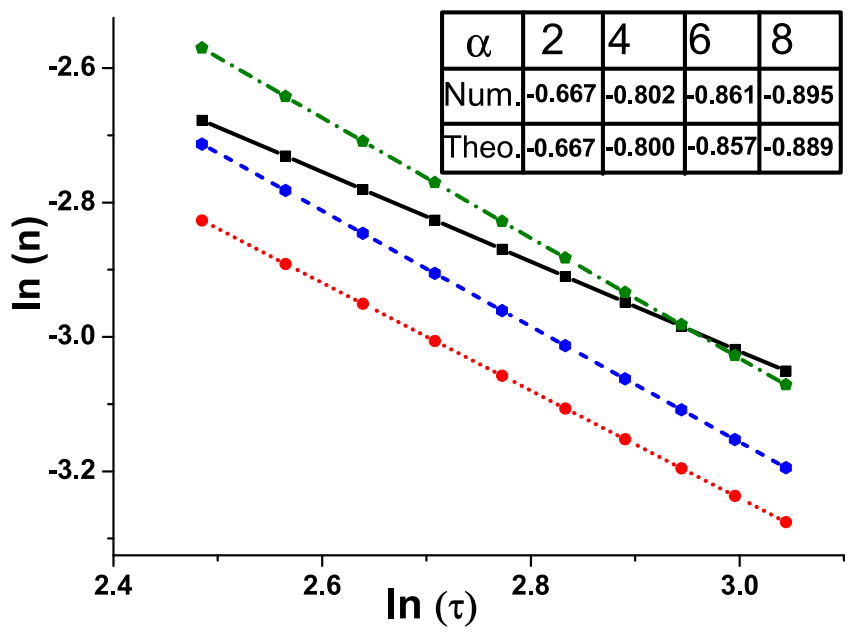

FIG. 3: Plot of $\ln (n)$ vs $\ln (\tau)$ for the one-dimensional Kitaev model for $\alpha=2$ (black solid line), $\alpha=4$ (red dotted line), $\alpha=6$ (blue dashed line) and $\alpha=8$ (green dash-dotted line). The slopes of these lines agree reasonably with the predicted theoretical values $-\alpha /(\alpha+1)$ as shown in the table.

curves gives the values of the exponents to be $0.506 \pm$ $0.006,0.504 \pm 0.004$ and $0.505 \pm 0.002$ for $\tau=10,15$ and 20 respectively which are remarkably close to the theoretical value $1 / 2$. The systematic positive deviation of the exponents from the theoretical value $1 / 2$ comes from the contribution of the higher order terms neglected in the derivation of Eqs. (23) and (24). We note that the region of validity of our linear expansion, as can be seen from Fig. 2, grows with $\tau$ which is in accordance with the result in Eq. (25).

Next, we consider the one-dimensional Kitaev model which is governed by the Hamiltonian in Eq. (52). As mentioned in Sec. 54, such a model can also be mapped on to the free fermionic Hamiltonian given by Eq. (53). This system passes through the quantum critical point at $J_{-}=0$ for $k=\pi / 2$ when $J_{-}(t)=J_{-}|t / \tau|^{\alpha} \operatorname{sign}(t)$ is varied nonlinearly with time. Here the quantum critical point is at $t=0$. Thus from Eq. (19) we expect the defect density $n \sim \tau^{-\alpha /(\alpha+1)}$ since $\nu=z=1$ for this system. To check this prediction, we numerically solve the Schrödinger equation $i \partial_{t} \psi(k)=H^{\prime}(k ; t) \psi(k, t)$ and compute the defect density $n=\int_{0}^{\pi} d k / \pi p_{k}$ as a function of the quench rate $\tau$ for different $\alpha$ with fixed $J_{+} / J=1$. A plot of $\ln (n)$ vs $\ln (\tau)$ for different values of $\alpha$ is shown in Fig. 3. The slope of these lines, as can be seen from Fig. 3] changes from -0.67 towards -1 as $\alpha$ increases from 2 towards larger values. This behavior is consistent with the prediction of Eq. (19). The slopes of these lines also show excellent agreement with Eq. (19) as shown in the inset of Fig. 3 .

Finally, we illustrate the expressions in Eqs. (27) and (32) by taking two one-dimensional models governed by Eqs. (11) and (26) with $z_{1}=2, z_{2}=1$ and $z_{1}=1, z_{2}=2$ respectively. Setting $\alpha=4$, we numerically carry out the time evolutions for different values of the momentum

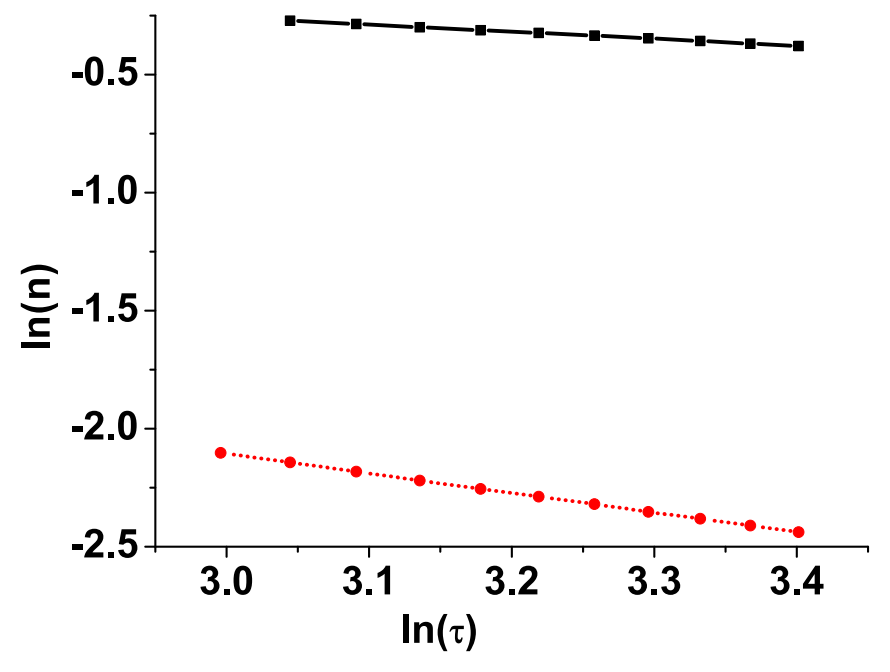

FIG. 4: Plots of $\ln (n)$ vs $\ln (\tau)$ for models with $z_{1}=2$ and $z_{2}=1$ (red dotted lower line) and $z_{1}=1$ and $z_{2}=2$ (black solid upper line), for $d=1$ and $\alpha=4$. The slopes of the lower and upper lines are -0.828 and -0.301 which compare reasonably with the predicted theoretical values of $-4 / 5=$ -0.8 and $-4 / 13=-0.308$ respectively.

$k$ and then integrate to compute the defect density as a function of $\tau$. The results are shown in Fig. 4, reasonable agreement is obtained with the theoretical values of the exponents given in Eqs. (27) and (32).

\section{EXPERIMENTS}

The generality of our results allows for their verification in several realizable experimental systems. We note that all our results have been obtained at zero temperature with the assumption that the system does not relax significantly during the quench process and till the measurement of the defect density has been performed. This might seem too restrictive. However, we would like to point out that systems of ultracold atoms in optical or magnetic traps and/or optical lattices can easily satisfy the required criteria since they have a very long relaxation time which often gets close to the system lifetime $\underline{27}$. We list some possible experiments briefly here. First, there has been a concrete proposal for the realization of the Kitaev model using an optical lattice ${ }^{28}$. In such a realization, all the couplings can be independently tuned using separate microwave radiations. In the proposed experiment, one needs to keep $J_{3}=0$ and vary $J_{1(2)}=J\left(1 \pm|t / \tau|^{\alpha} \operatorname{sign}(t)\right) / 2$, so that $J_{+}$remains constant while $J_{-}$varies in time. The variation of the defect density, which in the experimental set-up would correspond to the bosons being in the wrong spin state, would then show the theoretically predicted power law behavior in Eq. (19). Secondly, a similar quench experiment can be carried out with spin-1 bosons in a magnetic field described by an effective Hamiltonian 
$H_{\text {eff }}=c_{2} n_{0}\langle\mathbf{S}\rangle^{2}+c_{1} B^{2}\left\langle S_{z}^{2}\right\rangle \underline{29}$, where $c_{2}<0$ and $n_{0}$ is the boson density. Such a system undergoes a quantum phase transition from a ferromagnetic state to a polar condensate at $B^{*}=\sqrt{\left|c_{2}\right| n_{0} / c_{1}}$. A quench of the magnetic field $B^{2}=B_{0}^{2}|t / \tau|^{\alpha}$ would thus lead to a scaling of the defect density with an effective rate $\tau_{\text {eff }}(\alpha)=\tau /\left(\alpha g^{(\alpha-1) / \alpha}\right)$, where $g=\left|c_{2}\right| n_{0} / c_{1}$. A measurement of the dependence of the defect density $n$ on $\alpha$ should therefore serve as a test of the prediction in Eq. (24). Finally, spin gap dimer compounds such as $\mathrm{BaCuSi}_{2} \mathrm{O}_{6}$ are known to undergo a singlet-triplet quantum phase transition at $B_{c} \simeq 23.5 \mathrm{~T}$ which is known to be very well described by the mean-

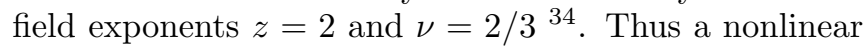
quench of the magnetic field through its critical value $B=B_{c}+B_{0}|t / \tau|^{\alpha} \operatorname{sign}(t)$ should lead to a scaling of the defects $n \sim \tau^{-6 \alpha /(4 \alpha+3)}$ in $d=3$. In the experiment, the defect density would correspond to residual singlets in the final state which can be computed by measuring the total magnetization of the system immediately after the quench. We note that for these dimer systems, it will be necessary to take special care to achieve the criterion of long relaxation time mentioned earlier.

\section{CONCLUSIONS}

In conclusion, we have studied defect production in quantum critical systems for an arbitrary nonlinear power law quench. We have shown that the defect production rate depends crucially on whether the system passes from one gapped phase to another or along a critical gapless line during the quench. We have obtained general scaling laws for defect densities produced during the quench for both these cases, and have verified these laws by numerical studies of one-dimensional systems. We have also computed the defect correlation functions for a class of $d$-dimensional models and have discussed the scaling of the amplitude of these functions with the quenching rate. Finally, we have discussed several experimental systems where these results can, in principle, be tested.

The authors thank A. Dutta and A. Polkovnikov for helpful comments and discussions. DS acknowledges financial support from DST, India under Project No. SR/S2/CMP-27/2006.
1 S. Sachdev, Quantum Phase Transitions (Cambridge University Press, Cambridge, 1999).

2 P. Gegenwart, Q. Si, and F. Steglich, Nature Phys. 4, 186 (2008).

3 S. Sachdev, Nature Phys. 4, 173 (2008).

4 T. Giamarchi, C. Ruegg, and O. Tchernyshyov, Nature Phys. 4, 198 (2008).

5 K. Damle and S. Sachdev, Phys. Rev. B 56, 8714 (1997); ibid Phys. Rev. Lett. 78, 943 (1997); S. A. Hartnoll, P. K. Kovtun, M. Mueller, and S. Sachdev, Phys. Rev. B 76, 144502 (2007); A. del Maestro, B. Rosenow, N. Shah, and S. Sachdev, Phys. Rev. B 77, 180501 (2008).

${ }^{6}$ K. Sengupta, S. Powell, and S. Sachdev, Phys. Rev. A 69, 053616 (2004).

7 T. W. B. Kibble, Phys. A 9, 1387 (1976).

8 W. H. Zurek, Nature (London) 317, 505 (1985).

9 B. Damski, Phys. Rev. Lett. 95, 035701 (2005).

10 J. Dziarmaga, Phys. Rev. Lett. 95, 245701 (2005); ibid Phys. Rev. B 74, 064416 (2006).

11 P. Calabrese and J. Cardy, J. Stat. Mech: Theory Expt P04010 (2005), and Phys. Rev. Lett. 96, 136801 (2006).

12 A. Das, K. Sengupta, D. Sen, and B. K. Chakrabarti, Phys. Rev. B 74, 144423 (2005).

13 R. W. Cherng and L. Levitov, Phys. Rev. A 73, 043614 (2006).

14 V. Mukherjee, U. Divakaran, A. Dutta, and D. Sen, Phys. Rev. B 76, 174303 (2007).

15 B. Damski and W. H. Zurek, Phys. Rev. A 73, 063405 (2006).

16 T. Caneva, R. Fazio, and G. E. Santoro, Phys. Rev. B 76, 144427 (2007).

17 F. M. Cucchietti, B. Damski, J. Dziarmaga, and W. H. Zurek, Phys. Rev. A 75, 023603 (2007).

18 K. Sengupta, D. Sen, and S. Mondal, Phys. Rev. Lett. 100, 077204 (2008); S. Mondal, D. Sen, and K. Sengupta, Phys.
Rev. B 78, 045101 (2008).

19 F. Pellegrini, S. Montangero, G. E. Santoro, and R. Fazio, arXiv:0801.4475 (unpublished).

20 U. Divakaran, A. Dutta, and D. Sen, arXiv:0805.3328 (unpublished).

21 U. Divakaran, V. Mukherjee, A. Dutta, and D. Sen, arXiv:0807.3606 (unpublished).

22 D. Patane, A. Silva, L. Amico, R. Fazio, and G. E. Santoro arXiv:0805.0586 (unpublished).

23 A. Polkovnikov, Phys. Rev. B 72, 161201(R) (2005).

24 A. Polkovnikov and V. Gritsev, arXiv:0706.0212 (unpublished).

25 D. Sen, K. Sengupta, and S. Mondal, Phys. Rev. Lett. 101, 016806 (2008).

26 R. Barankov and A. Polkovnikov, arXiv:0804.2894 (unpublished).

27 For a review, see I. Bloch, J. Dalibard, and W. Zwerger, arXiv:0704.3011, to appear in Rev. Mod. Phys.

28 L.-M. Duan, E. Demler, and M. D. Lukin, Phys. Rev. Lett. 91, 090402 (2003); A. Micheli, G. K. Brennen, and P. Zoller, Nature Physics 2, 341 (2006).

29 L. E. Sadler, J. M. Higbie, S. R. Leslie, M. Vengalattore, and D. M. Stamper-Kurn, Nature (London) 443, 312 (2006).

30 See for example, L. Landau and E. M. Lifshitz, Quantum Mechanics: Non-relativistic Theory, 2nd Ed. (Pergamon Press, Oxford, 1965); S. Suzuki and M. Okada in Quantum Annealing and Related Optimization Methods, Eds. by A. Das and B. K. Chakrabarti (Springer-Verlag, Berlin, 2005).

31 H.-D. Chen and Z. Nussinov, J. Phys. A 41, 075001 (2008).

32 D.-H. Lee, G.-M. Zhang, and T. Xiang, Phys. Rev. Lett. 99, 196805 (2007).

33 A. Kitaev, Ann. Phys. 321, 2 (2006).

34 S. E. Sebastian, P. A. Sharma, M. Jaime, N. Harrison, V. Correa, L. Balicas, N. Kawashima, C. D. Batista, and I. 
R. Fisher, Phys. Rev. B 72, 100404(R) (2005). 